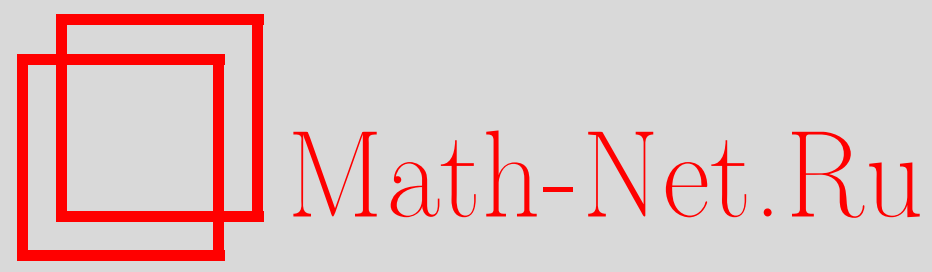

А. Р. Чехлов, Квазисервантно инъективные группы без кручения с неразложимыми сервантными подгруппами, Матем. заметки, 2000, том 68, выпуск 4, 587-592

DOI: https://doi.org/10.4213/mzm979

Использование Общероссийского математического портала Math-Net.Ru подразумевает, что вы прочитали и согласны с пользовательским соглашением http://www . mathnet.ru/rus/agreement

Параметры загрузки:

IP : 54.198 .55 .26

26 апреля 2023 г., $15: 10: 35$

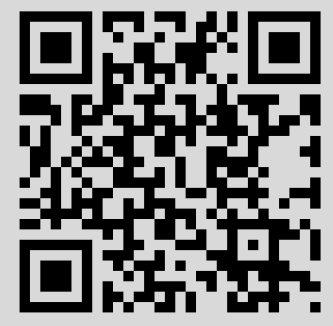




\section{КВАЗИСЕРВАНТНО ИНЪЕКТИВНЫЕ ГРУППЫ БЕЗ КРУЧЕНИЯ С НЕРАЗЛОЖИМЫМИ СЕРВАНТНЫМИ ПОДРРУППАМИ}

\section{А.Р. Чехлов}

Показано, что в абелевой квазисервантно инъективной группе без кручения с сильно неразложимыми сервантными подгруппами все ненулевые эндоморфизмы являются мономорфизмами. Результаты статьи вместе с полученными ранее описьвают квазисервантно инъективные группы без кручения. В качестве следствия доказьвается, что квазисервантно инъективная группа без кручения транзитивна.

Библиографоия: 10 названий.

Абелева группа $A$ называется квазисервантно инвективной (сокращенно $Q P I$ группой), если всякий гомоморфизм $G \rightarrow A$ любой ее сервантной подгруппы $G$ продолжается до эндоморфизма группы $A$. Исследованию $Q P I$-групп посвящены работы ряда авторов (см., например, [1]-[8]). Обозначим через $L$ класс всех редуцированных групш без кручения, тип любого ненулевого элемента которых сравним с некоторым максимальным типом в множестве всех ненулевых элементов групп. Класс $L$ содержит все группы без кручения конечного ранга, однородные группы без кручения, алгебраически компактные групшы без кручения и др. В [1], [2] дано структурное описание $Q P I$-групп без кручения из класса $L$. Независимо $Q P I$-группы без кручения конечного ранга были описаны в [3]. В [4] построены примеры $Q P I$-групп, не принадлежаших $L . \quad$ В [5] доказано, что редуцированная $Q P I$-группа без кручения является сервантной вполне характеристической межпрямой суммой своих квазиоднородных компонент, каждая из которых либо однородная группа, либо группа с сильно неразложимьми сервантными подгруппами. В [6] получена характеризация квазиоднородных $Q P I$-груп, все ненулевые эндоморфизмы которых являются мономорфизмами. В [8] дан обзор результатов о $Q P I$-группах и близких к ним классов групп.

В данной работе показано, что у квазиоднородной $Q P I$-группы с сильно неразложимыми сервантньми подгруппами все ненулевые эндоморфизмы являются мономорфизмами. Таким образом, результаты статьи завершают описание $Q P I$-групп без кручения (см. [9, проблема 17 a)]).

Терминология и обозначения соответствуют [9], [10]. Так, если $A$ - групша без кручения, то $h_{p}^{A}(a)$ - p-высота, $\chi_{A}(a)$ - характеристика, $t_{A}(a)$ - тип элемента $a$ в групе $A$; если $G \subseteq A$, то $\langle G\rangle_{*}^{A}$ - сервантная подгруппа в $A$, порожденная $G$ (индекс $A$ иногда опускаем); $A(t)=\{a \in A \mid t(a) \geqslant t\} ; \Pi(A)$ - множество всех простых чисел $p$ со свойством $p A \neq A ; E(A)$ - кольцо эндоморфизмов $A$; если $p$-простое число, то $p$ - $p$ ангом $A$ называется ранг ее факторгрушы $A / p A ; T(A)$ - множество типов всех ненулевых элементов 
группы $A$. Группа без кручения $A$ называется квазиоднородной, если $\Pi(G)=\Pi(A)$ для любой ее ненулевой сервантной подгруппы $G$; вполне транзитивной, если для любых ее элементов $a, b$ условие $\chi_{A}(a) \leqslant \chi_{A}(b)$ влечет существование ее эндоморфизма $f$ со свойством $f(a)=b ;$ квазиразложимой, если существуют ее ненулевые подгрупшы $B, G$ такие, что $n A \subseteq B \oplus G$ для некоторого натурального числа $n$, в противном случае $A$ назьвается сильно неразложимой. Отметим, что все $Q P I$-группы без кручения являются вполне транзитивньми. Свойство, что все ненулевые эндоморфизмы есть мономорфизмы для $Q P I$-группы $A$ без кручения эквивалентно тому, что для любых ее элементов $0 \neq a, b, c$ вьполнено условие $t_{A /\langle a\rangle_{*}}\left(b+\langle a\rangle_{*}\right) \nless t_{A}(c)$.

ЛЕмма 1 [2]. Пусть $A$ - группа без кручения с сильно неразложсимыми сервантными подгруппами. Тогда $t_{A /\langle a\rangle_{*}}\left(g+\langle a\rangle_{*}\right)>t_{A}(g)$ для любых ее ненулевых әлементов $a, g$. В частности, если $f$ - гомоморфизм $A$ в группу без кручения $B$ с ненулевым ядром, то $t_{A}(g)<t_{B}(f(g))$ для любого $g \in A$.

ДоказАтЕльство. Сильная неразложимость подгрупшы $\langle a, g\rangle_{*}$ в $A$ влечет разрешимость в $A$ уравнения $m x=b+g$, где $b \in\langle a\rangle_{*}$ и $g \notin m A$ для бесконечного множества натуральных $m$. Отсюда вытекает справедливость указанного неравенства типов.

Лемма 2. Пусть $A$ - группа без кручения, $f \in E(A), 0 \neq b \in \operatorname{Ker} f$. Тогда выполнены следующие утверждения:

1) если $h_{p}(b) \geqslant h_{p}(f(a))$, то $h_{p}(a+b)=h_{p}(a)$; в частности, если $\chi(b) \geqslant \chi(f(a))$, mo $\chi(a+b)=\chi(a)$

2) если $A$ вполне транзитивна, то $f(A)$ содержсится в замыкании в $p$-адической топологии группы $A$ ее подгруппы $G=A(t(b))$ для каждого $p \in \Pi(G)$; в частности, если $\Pi(A)=\Pi(G)$, то $f(A)$ содержится в замыкании в $Z$-адической топологии подгруппы $G$.

ДоказАТЕЛьство. 1) Имеем $h_{p}(a+b) \geqslant h_{p}(a) \cap h_{p}(b)=h_{p}(a)$. Далее $h_{p}(a+b) \leqslant$ $h_{p}(f(a+b))=h_{p}(f(a)) \leqslant h_{p}(b)$, поэтому $h_{p}(a)=h_{p}((a+b)-b) \geqslant h_{p}(a+b) \cap h_{p}(b)=$ $h_{p}(a+b)$. Следовательно, $h_{p}(a)=h_{p}(a+b)$.

2) Если $p^{m} g=b$, где $g \notin p A$, то для любого $a \in A$ и любого натурального $n$ имеем $\chi\left(p^{n} a+g\right) \leqslant \chi(f(a))$. В силу вполне транзитивности существует эндоморфизм $\alpha$ со свойством $p^{n} \alpha(a)+\alpha(g)=f(a)$. Утверждение леммы следует из того, что $\alpha(g) \in G$.

Лемма 3. Пусть $A-$ - редуиированная вполне транзитивная группа без кручения, $f \in E(A)$ и $G=A(t) \subseteq \operatorname{Ker} f$ для некоторого $t \in T(A)$, причем $\Pi(G)=\Pi(A)$. Тогда $f=0$.

ДокАЗАТЕЛЬСТво. Из леммы 2 следует, что $f^{2}(A)$ является делимой подгруппой. В силу редуцированности $f^{2}=0$. Предположим, что $f(a) \neq 0, a \in A$. Согласно лемме 2 $\chi(a+f(a))=\chi(a)$. Тогда $\chi\left(p^{n} a+g\right) \leqslant \chi(a)$, где $p^{m} g=f(a), g \in A \backslash p A, n>m$ и $p \in \Pi(A)$. По лемме $2 f(a)$ принадлежит замьканию в $p$-адической топологии подгруппы $G$. В силу сервантности $G$ это замькание является сервантной подгруппой в $A$. Поэтому $g$ можно записать в виде $g=x+p^{n} b$ для некоторых $x \in G, b \in A$. Пусть $\alpha \in E(A)$ такой, что $p^{n} \alpha(a+b)+\alpha(x)=a$. Так как $\alpha(x) \in G \subseteq \operatorname{Ker} f$, то $p^{n} f(\alpha(a+b))=f(a)$, что противоречит выбору $n$. Следовательно, $f=0$. 
Согласно [5, теорема 2.1] неквазиоднородная $Q P I$-группа без кручения разложима. Поэтому $Q P I$-группы без кручения с неразложимыми сервантными подгруппами квазиоднородны.

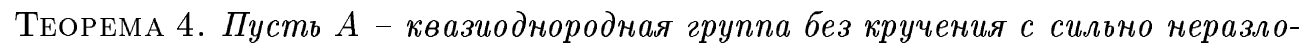
жимыми сервантными подгруппами и с условием обрыва возрастающих иепей ядер ее әндоморфизмов. Тогда если $А$ вполне транзитивна, то все ее ненулевые әндоморфизмы являются мономорфизмами.

ДокАЗАтЕЛЬСтво. Пусть $f \in E(A)$ с максимальным ядром $G \neq 0$ и $f(a) \neq 0, a \in A$. Из леммы 3 следует, что $f(b) \neq 0$ для некоторого $b \in A(t(f(a)))$. Тогда $\chi(n b) \geqslant \chi(f(a))$ для некоторого натурального $n$ и $\alpha f(a)=n b$, где $\alpha \in E(A)$. Отсюда следует, что $(\alpha f)^{2} \neq 0$. Будем считать, что $f^{2} \neq 0$, в противном случае в качестве $f$ возьмем $\alpha f$.

По лемме 1 найдется $p \in \Pi(A)$ со свойством $h_{p}^{A / G}(f(a)+G)>h_{p}^{A}(f(a))$. Отсюда $m=h_{p}\left(f^{2}(a)\right)-h_{p}(f(a))>0$. Имеем $p^{m} \varphi f(a)=f^{2}(a)$ для некоторого $\varphi \in E(A)$. Так как $\langle G, a\rangle \subseteq \operatorname{Ker}\left(f-p^{m} \varphi\right) f$, то $f^{2}=p^{m} \varphi f$. Следовательно, в силу квазиоднородности для каждого $p \in \Pi(A)$ найдутся целое число $n \geqslant 0$ и эндоморфизм $\beta$ такие, что $f^{2}=p^{n} \beta$, где $\beta$ сохраняет $p$-высоты элементов из $f(A)$ и, следовательно, элементов из $B=\langle f(A)\rangle_{*}$.

Если теперь $p x=b+g$, где $p \in \Pi(A), b \in B, g \in G$, то $p \beta(x)=\beta(b)$. Откуда $b \in p B$ в силу условия на $\beta$. Это влечет сервантность подгруппы $B \oplus G$ в $A$, что противоречит условию теоремы.

СлЕдСТВИЕ 5. Если $A$ - квазиоднородная вполне транзитивная группа без кручения конечного р-ранга для некоторого $p \in \Pi(A)$ с сильно неразложимыми сервантными подгруппами, то все ее ненулевые әндоморфизмы являются мономор$\oint и з м а м и$.

ДокАЗАТЕЛЬСТво. В силу квазиоднородности я́дра эндоморфизмов группы $A$ являются замкнутыми сервантными подгруппами в ее $p$-адической топологии. Поэтому конечность $p$-ранга влечет условие обрьва возрастающих цепей ядер ее эндоморфизмов, что доказьвает справедливость утверждения.

Теорема 6. Если $A$ - квазиоднородная QPI-группа без кручения, имеющая ненулевые әндоморфизмы с ядрами бесконечного р-ранга для некоторого простого числа $p$, то $A$ - однородная алгебраически компактная группа.

ДоказАТЕЛьСтво. Пусть $B=\operatorname{Ker} f, p$-ранг $B$ бесконечен и $F-p$-базисная подгруппа в $B$. Так как $F-$ свободная группа бесконечного ранга, то в $F$ найдется подгруппа $K$ такая, что $F / K$ - редуцированная группа без кручения ранга $1, q$-делимая для каждого простого $q \neq p$. Поскольку замыкание $F^{-}$в $p$-адической топологии $A$ $p$-сервантной подгруппы $F$ является сервантной подгруппой, то $H=K^{-} \subset F^{-}$и, так как $F \nsubseteq H$, то факторгрупша $F^{-} / H$ содержит сервантную подгруппу $G / H \cong F / K$. Пусть $b+H \in(G / H) \backslash p(G / H)$. Предположим, что $f(a) \neq 0$ для некоторого $a \in A \backslash p A$. В силу квазиоднородности $n=h_{p}(f(a))<\infty$. Пусть $D=\left\langle p^{k} a+b, H\right\rangle_{*}$, где $k>n$. Тогда $D / H$ - группа ранга 1 и $\chi_{A / H}\left(p^{k} a+b+H\right) \leqslant \chi_{A}(f(a))$. Если $\pi: D \rightarrow D / H-$ естественный эпиморфизм, а $\psi: D / H \rightarrow\langle f(a)\rangle_{*}$ - такой гомоморфизм, что $\psi\left(p^{k} a+b+H\right)=f(a)$, то так как $A-Q P I$-группа, $\psi \pi$ продолжается до некоторого $\alpha \in E(A)$. Имеем 
$\alpha\left(p^{k} a+b\right)=f(a)$, причем $\alpha(b) \neq 0$, так как $f(a) \notin p^{k} A$. Подгруппа $\langle\alpha(b)\rangle_{*} q$-делима для каждого простого $q \neq p$. В силу квазиоднородности группа $A$ также $q$-делима для всех таких $q$. Следовательно, $A$ - однородная $Q P I$-группа бесконечного $p$-ранга. Из [1, теорема 1.4] следует, что она алгебраически компактна.

ТЕОрема 7. Если $A$ - квазиоднородная QPI-группа без кручения с сильно неразложимыми сервантными подгруппами, то все ненулевые әндоморфизмы $А$ являются мономорфизмами.

ДокАЗАТЕЛЬСтво. Если $A$ однородна, то это утверждение доказано в $[1$, теорема 1.4]. Пусть она неоднородна. Зафиксируем $p \in \Pi(A)$ и пусть $f(a)=0$, где $a \in A \backslash p A$ и $0 \neq f \in E(A)$. Тогда $\langle a\rangle-p$-сервантная подгруппа в $A$ и ее можно вложить в $p$-базисную подгруппу $B$ групшы $A$ в качестве прямого слагаемого, $B=\langle a\rangle \oplus G[9, \S 32$, упр. 7]. Если $\bar{A}-p$-адическое пополнение $A$, то $\bar{A}=R \oplus \bar{G}$, где $R=\overline{\langle a\rangle}$. В силу $p$-сервантной инъективности $f$ продолжается до $\bar{f} \in E(\bar{A})$. Ввиду следствия $5 p$-ранг $A$ бесконечен, поэтому $G$ является свободной групой бесконечного ранга. Выделим в $G$ такое прямое слагаемое $H$ счетного ранга, что $G=H \oplus D$ и ранг $D$ бесконечен. Имеем $H=\bigoplus_{i=1}^{\infty}\left\langle b_{i}\right\rangle$. $\Gamma$ руппа $R$ имеет ранг мощности континуум, выделим в ней счетную линейно независимую систему элементов $g_{i}(i=1,2, \ldots)$. Отображение $b_{i} \rightarrow g_{i}$ продолжается до гомоморфизма $H \rightarrow R$. Так как $R$ - $p$-сервантно инъективная группа, а $H-p$-сервантная подгруппа, то этот гомоморфизм продолжается до гомоморфизма $\varphi: \bar{H} \rightarrow R$. Если $\pi$ и $\theta$ - проекции $M=R \oplus \bar{H}$ на $R$ и $\bar{H}$ соответственно, то $M=R \oplus K$, где $K=(\theta-\pi \varphi \theta) M[9$, лемма 9.5]. Имеем $b_{i}=(\pi+\pi \varphi \theta)\left(b_{i}\right)+(\theta-\pi \varphi \theta)\left(b_{i}\right)=\varphi\left(b_{i}\right)+\left(b_{i}-\varphi\left(b_{i}\right)\right)$, где $b_{i}-\varphi\left(b_{i}\right) \in K$. Следовательно, $H \cap K=\langle H\rangle_{*}^{A} \cap K=0$. В силу бесконечности ранга $D$ из теоремы 6 следует, что найдется $g \in D \backslash p D$ со свойством $f(g) \neq 0$. Имеем $\bar{D}=\overline{\langle g\rangle} \oplus C$ и $\bar{A}=(R \oplus \overline{\langle g\rangle}) \oplus K \oplus C$. Если $\alpha-$ проекция $\bar{A}$ на $R \oplus \overline{\langle g\rangle}$, то $(\bar{f} \alpha)\left(\langle H, g\rangle_{*}^{A}\right) \subseteq\langle f(g)\rangle_{*}^{A} \subseteq A$. Так как $A-Q P I$-группа, то этот гомоморфизм продолжается до ее эндоморфизма, ядро которого содержит $H$ и, следовательно, имеет бесконечньй $p$-ранг. Полученное противоречие с теоремой 6 заканчивает доказательство.

Для полноты приведем следующий результат.

Tеорема 8 [7, теорема 2]. Пусть квазиоднородная QPI-группа без кручения $A$ имеет квазиразложимые сервантные подгруппы. Тогда она однородна.

ДокАЗАТЕЛЬСтво. По условию $A$ содержит сервантную подгруппу $B \neq 0$ со свойством $n\langle a, B\rangle_{*} \subseteq\langle a\rangle_{*} \oplus B$, где $n$ натуральное и $0 \neq a \in A$. Зафиксируем $a$ и $n$. Множество $M$ всех таких сервантных подгрупп частично упорядочено относительно включения и индуктивно, поэтому оно содержит максимальный элемент $G$. Пусть $\bar{A}$ есть $\mathbb{Z}$-адическое пополнение $A$. Имеем $\bar{A}=\langle\bar{F}\rangle_{*} \oplus V$, где $F=\langle a\rangle_{*} \oplus G$. Далее, $n\langle\bar{F}\rangle_{*} \subseteq \bar{W} \oplus \bar{G}=\bar{F}, n \bar{A} \subseteq \bar{F} \oplus V=N$, где $W$ - замькание в $\mathbb{Z}$-адической топологии группы $A$ ее подгруппы $\langle a\rangle_{*}$. Отметим, что $W=A \cap \bar{W}$. Так как $G \subseteq\langle A \cap(\bar{G} \oplus V)\rangle_{*} \in M$, то $A \cap(\bar{G} \oplus V)=G$ в силу максимальности $G$.

Пусть $\theta$ - проекция $N$ на $V$. Тогда $\theta(D)$ - сервантная подгруппа в $V$, где $D=A \cap N$ сервантна в $N$. Действительно, если $m x=v \in \theta(D)$, то $b+v \in D$ для некоторого $b \in \bar{F}$. Так как $F$ плотна в $\bar{F}$, то $b-d \in m \bar{F}$ для некоторого $d \in F$. Имеем $(b-d)+v \in D \cap m N=m D$. Поэтому $(b-d)+v=m y, y \in D$. Откуда $m \theta(y)=x$. 
Из [1, теорема 1.6] вытекает, что квазиоднородная $Q P I$-группа, имеющая максимальный тип в $T(A)$, принадлежит классу $L$ (см. также [7, лемма 2]). По теореме 5.1 из [2] $Q P I$-группа из $L$ с квазиразложимыми сервантными подгрупшами однородна. Поэтому предположим, что $A$ не имеет ненулевых элементов максимальных типов.

Пусть $t \in T(A)$ такой, что $t>t(a), t(g)$, где $0 \neq g \in G$. Такой $t$ существует. Действительно, пусть $p \in \Pi(A)$ не делит $n$. Можно считать, что $h_{p}(a)=0$. Так как $\chi_{A}(p n a+g) \leqslant \chi_{A}(n a)$, то существует $\psi \in E(A), \psi(p n a+g)=n a$. Имеем $0 \neq \psi(g)=n a-p \psi(n a) \in A$. Откуда $t(\psi(g)) \geqslant t(g), t(a)$. Существование $t$ вытекает из условия на типы элементов.

Допустим, что $A(t) \cap W=0$. Если $\pi$ - проекция $F$ на $\langle a\rangle_{*}$, то $\pi n$ продолжается до $\varphi \in E(A)$, которьй в силу сервантной инъективности можно считать эндоморфизмом $\bar{A}$. Так как $G$ плотна в $\bar{G}$, то $\varphi(\bar{G})=0$, по аналогичной причине $\varphi$ действует на $\bar{W}$ как умножение на $n$. Поэтому если $A(t) \subseteq\langle\bar{F}\rangle_{*}$, то $\varphi(A(t)) \subseteq A(t) \cap W=0$. По лемме 2 $\varphi=0$. Противоречие. Следовательно, найдется $0 \neq x=b+g+v \in D(t)$, где $b \in \bar{W}$, $g \in \bar{G}, 0 \neq v \in V$. Если $b=0$, то $G \subset\langle G, g+v\rangle_{*} \in M$. Поэтому $b \neq 0$. Так как $\theta(D(t))$ сервантна в $V$, то если $v=m v_{1}, v_{1} \in V$, существует $y=b_{1}+g_{1}+v_{1} \in D(t)$, где $b_{1} \in \bar{W}$, $g_{1} \in \bar{G}$. Откуда $x-m y=\left(b-m b_{1}\right)+\left(g-m g_{1}\right)$ и $\varphi(x-m y)=n\left(b-m b_{1}\right) \in A(t) \cap W=0$. Следовательно, $b=m b_{1}$ и, значит, $\chi(b) \geqslant \chi(v)$. Так как $\bar{W}$ сервантно инъективна, то существует гомоморфизм $f: V \rightarrow \bar{W}$ со свойством $f(v)=-b$. Тогда $N=\bar{F} \oplus V_{1}$, где $b+v \in V_{1}\left[9\right.$, лемма 9.5]. Откуда $G \subset\langle G, x\rangle_{*} \in M$, что противоречит максимальности $G$. Итак, $W \cap A(t) \neq 0$ и $G \cap A(t) \neq 0$.

Таким образом, найдутся $0 \neq y \in G$ и $0 \neq x \in W$ со свойствами $t(x)>t(y)>t(a)$, $\chi(x)>\chi(y)>\chi(a)$. Рассмотрим подгруппу $E=\langle x, a+y\rangle_{*}^{N}$. Имеем $\chi(\bar{x}) \leqslant \chi_{N}(x)$, где $\bar{x}=x+\langle a+y\rangle_{*}^{N}-$ смежньй класс. Действительно, это вытекает из того, что для любого простого числа $q$ и любого рационального числа $r$ вьполняется $h_{q}^{N}(x+r(a+y)) \leqslant$ $h_{q}^{N}(r y), h_{q}^{N}(x+r a)$. Поэтому если $\delta: E \rightarrow E /\langle a+y\rangle_{*}^{N}-$ естественный эпиморфизм на группу ранга 1 , то существует гомоморфизм $\alpha: E /\langle a+y\rangle_{*}^{N} \rightarrow\langle x\rangle_{*}^{A}$ со свойством $\alpha(\bar{x})=x$. Имеем $\alpha \delta n(x)=n x$, где $\alpha \delta n:\langle E\rangle_{*}^{A} \rightarrow\langle x\rangle_{*}^{A}$. Следовательно, существует $\beta \in E(A)$, продолжающий $\alpha \delta n$, он действует на $W$ как умножение на $n$. Из $a+y \in \operatorname{Ker} \beta$ следует, что $\beta(a)=n a=-\beta(y)$. Отсюда $t(a)=t(\beta y) \geqslant t(y)$. Полученное противоречие доказьвает теорему.

Напомним, что группа без кручения $A$ назьвается транзитивной, если для любых ее элементов $0 \neq a, b$ условие $\chi_{A}(a)=\chi_{A}(b)$ влечет существование ее автоморфизма $f$ со свойством $f(a)=b$. Из полученных результатов следует, что вопрос 1 из $[8, \S 6]$ имеет положительное решение.

\section{СлЕДСТВИЕ 9. QPI-группа без кручения является транзитивной.}

ДокАЗАТЕЛЬСтво. Нередуцированная группа без кручения транзитивна тогда и только тогда, когда транзитивна ее редуцированная часть. Редуцированная $Q P I$-группа без кручения является сервантной вполне характеристической межпрямой суммой своих квазиоднородных компонент [5, теорема 2.1]. Вопрос об ее транзитивности сводится к вопросу о транзитивности квазиоднородных компонент. Если квазиоднородная $Q P I-$ группа однородна, то она транзитивна [2, следствие 3.3]. По теореме 7 в неоднородной $Q P I$-группе $A$ все ненулевые эндоморфизмы - мономорфизмы, поэтому $A$ транзитивна. 
Действительно, если $\chi(a)=\chi(b)$, где $0 \neq a, b \in A$, и $\varphi, \psi \in E(A)$ такие, что $\varphi(a)=b$, $\psi(b)=a$, то $\psi \varphi(a)=a, \varphi \psi(b)=b$. Отсюда $\psi \varphi=\varphi \psi=1$, т.е. $\varphi$ и $\psi$ - автоморфизмы.

\section{СПИСОК ЦИТИРОВАННОЙ ЛИТЕРАТУРЫ}

[1] Добрусин Ю.Б. Квазисервантно инъективные группы // Абелевы группы и модули. Томск, 1979. С. 45-63.

[2] Добрусин Ю. Б. Квазисервантно инъективные абелевы группы без кручения // Абелевы группы и модули. Томск, 1980. С. 45-69.

[3] Arnold D.M., O'Brien, Reid J.D. Quasi-pure injective and projective torsion-free abelian groups of finite rank // Proc. London Math. Soc. 1979. V. 38. № 3. P. 532-544.

[4] Крылов П. А. Некоторые примеры квазисервантно инъективных и транзитивных абелевых групп без кручения // Абелевы группы и модули. № 7. Томск, 1988. С. 81-99.

[5] Чехлов А.Р. Квазисервантно инъективные абелевы группы без кручения // Матем. заметки. 1989. Т. 46. № 3. С. 93-99.

[6] Чехлов А. Р. Абелевы группы без кручения конечного $p$-рангас дополняемыми замкнутыми сервантными подгруппами // Абелевы группы и модули. № 10. Томск, 1991. С. 157-178.

[7] Чехлов А.Р. Об абелевых $Q C S$-группах без кручения // Абелевы группы и модули. № 11, 12. Томск, 1994. С. 240-245.

[8] Беккер И. Х., Крылов П. А., Чехлов А. Р. Абелевы группы без кручения, близкие к алгебраически компактным // Абелевы группы и модули. №11, 12. Томск, 1994. С. 3-52.

[9] Фукс Л. Бесконечные абелевы группы. Т. 1. М.: Мир, 1974.

[10] Фукс Л. Бесконечные абелевы группы. Т. 2. М.: Мир, 1977.

Томский государственный университет

Поступило

10.06 .1998

Исправленный вариант

21.03.2000 